\title{
RIESZ AND LAURENT DECOMPOSITIONS ON RIEMANN SURFACES
}

\author{
E. L. STOUT ${ }^{1}$
}

Introduction. This paper is motivated by a paper of Heins [3] in which a classical theorem of M. Riesz was generalized to a Riemann surface setting. The theorem of Riesz in question states that if $f$ is of class $L^{p}$ on the unit circle, $1<p<\infty$, then $f$ admits an essentially unique decomposition of the form

$$
f=f_{i}^{*}+f_{i}^{*}
$$

where $f_{i}^{*}$ is the boundary value function of a function of class $H^{p}$ on the unit disc and $f_{e}^{*}$ is the boundary value function of a function of class $H^{p}$ in the complement (in the Riemann sphere) of the closed unit disc. Heins showed that if $R$ is a compact bordered Riemann surface with double $\hat{R}$, then each $f \in L^{p}(\partial R), 1<p<\infty$, admits a decomposition of the form

$$
f=f_{i}^{*}+f_{e}^{*}+s
$$

where $f_{i}$ is of class $H^{p}$ on $R, f_{e}$ is of class $H^{p}$ on $\hat{R} \backslash R$, and $s$ is an element of a certain specified finite dimensional space of functions meromorphic on $\hat{R}$. The proof Heins gave for this result depends explicitly on the symmetry of the geometric configuration. In \$II below we will obtain a generalization of this result, a generalization which requires no particular symmetry in the ambient compact surface. We will also establish a theorem which is an analogue of Laurent's theorem valid on compact Riemann surfaces. Our result contains as a special case a result of Alling [1]. $\S I$ is devoted to certain algebraic preliminaries needed in the subsequent analytic considerations.

I. Algebraic preliminaries. We begin by setting some notation. If $S$ is a Riemann surface, let $\mathfrak{T}(S)$ and $\mathcal{O}(S)$ denote respectively the field of meromorphic functions on $S$ and the algebra of holomorphic

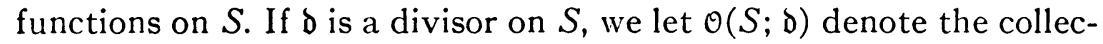
tion of elements $f$ of $\mathfrak{T}(S)$ whose divisors $(f)$ satisfy $(f) \geqq \delta$. If $\boldsymbol{b}$ is the unit divisor, the spaces $\mathcal{O}(S)$ and $\mathcal{O}(S ; \oslash)$ coincide.

The basic tool we will use in the sequel is a simple consequence of the Riemann-Roch theorem and the following result: Every principal

Received by the editors March 10, 1969.

1 The research for this note was supported by NSF Grant GP-9049. 
C-bundle on a compact Riemann surface has a nontrivial meromorphic section. Explicitly, if $R$ is a compact Riemann surface, if $\mathcal{u}=\left\{U_{i}\right\}_{i \in I}$ is an open cover for $R$, and if for $i, j \in I$ the functions $f_{i, j} \in \mathcal{O}\left(U_{i} \cap U_{j}\right)$ satisfy $f_{i, j}+f_{j, k}+f_{k, i}=0$ on $U_{i} \cap U_{j} \cap U_{k}$, then there exist functions $f_{i} \in \mathbb{M}\left(U_{i}\right)$ such that

$$
f_{i, j}=f_{i}\left|\left(U_{i} \cap U_{j}\right)-f_{j}\right|_{i}\left(U_{i} \cap U_{j}\right) .
$$

This result is derived, e.g., in [4, pp. 658-659].

Suppose now that we have an open covering $\mathcal{u}=\left\{U_{i}\right\}_{i \in I}$ of $R$ and a base point $P \in R$. Let the genus of $R$ be $g$. Assume that $f_{i, j} \in \mathcal{O}\left(U_{i} \cap U_{j}\right)$ and $f_{i} \in \mathfrak{M}\left(U_{i}\right)$ are related by (1.1). Since $f_{i}$ and $f_{j}$ differ on $U_{i} \cap U_{j}$ by a holomorphic function, it follows that the set $\Sigma$ of points in $R$ at which at least one of the functions $f_{i}$ has a pole is finite. Moreover, if $Q$ is a point of $\Sigma, Q \in U_{i} \cap U_{j}$, then the principal part of $f_{i}$ at $Q$ coincides with the principal part of $f_{j}$ at $Q$. Suppose $\Sigma=\left\{Q_{1}, \cdots, Q_{\mu}\right\}$, and for each $\nu=1, \cdots, \mu$, let $p_{\nu}$ be the order of the pole at $Q_{\nu}$ of those functions $f_{j}$ for which $U_{j}$ contains $Q_{\nu}$. We know that $p_{\nu}$ is a welldefined positive integer. The Riemann-Roch theorem implies that there is an $F \in \mathscr{T}(R)$ whose divisor is not less than the divisor $P^{-o} Q_{1}^{-p_{1}}$ $\cdots Q_{\mu}^{-p_{\mu}}$ and which has the property that the function $\tilde{f}_{i}=f_{i}-F \mid U_{i}$ is holomorphic on $U_{i}$ except for a possible pole, that of order no more than $g$, at $P$. We have that $\tilde{f}_{i} \in \mathcal{O}\left(U_{i} ; P^{-o}\right)$ and

$$
f_{i, j}=\tilde{f}_{i}\left|\left(U_{i} \cap U_{j}\right)-\tilde{f}_{j}\right|\left(U_{i} \cap U_{j}\right) .
$$

It is in this form that we will use the result. (Note that this statement admits the equivalent formulation that the cohomology group $H^{1}\left(R ; \mathcal{O}_{R} ; P^{-o}\right)=0$ if $\mathcal{O}_{R ; P^{-o}}$ is the sheaf of germs of functions whose divisors are not less than the divisor $P^{-\sigma}$.)

II. The Main Results. For our first application of the material of the last section, we consider a compact Riemann surface $R$ of genus $g$ and in $R$ a connected domain $G$ with nonempty boundary $\Gamma$. We suppose $\Gamma$ to consist of a finite collection of pairwise disjoint simple closed curves and that it is the boundary of $R \backslash \bar{G}$. We denote by $G_{1}$, $\cdots, G_{q}$ the components of $R \backslash \bar{G}$, and we set $X_{j}=R \backslash \bar{G}_{j}$. Let $Q_{1}$, $\cdots, Q_{q}$ be points of $G_{1}, \cdots, G_{q}$ respectively. In this situation, we have the following analogue of Laurent's theorem: ${ }^{2}$

II.1. THEOREM. Every $f \in \mathcal{O}(G)$ has a decomposition of the form

$$
f=f_{1}+\cdots+f_{q}+s
$$

2 The referee observed that in my original formulation of this theorem the domain $G$ was insufficiently restricted. 
where $f_{j} \in \mathcal{O}\left(X_{j}\right)$ and $s \in \mathcal{O}\left(R ; Q_{1}^{-g} \cdots Q_{q}^{-g}\right)$. If the points $Q_{1}, \cdots, Q_{q}$ are non-Weierstrass points, this decomposition is unique provided we require $f_{j}(P)=0$ for some fixed point $P \in G$ and $j=1, \cdots, q$.

Let us preface the proof of this theorem with a few remarks. In the case that $R$ is the Riemann sphere and $G$ is an annular domain, $q=2$ and $g=0$ so $\mathcal{O}\left(R ; Q_{1}^{-g} Q_{2}^{-g}\right)=C$, and we have the decomposition provided by Laurent's theorem in elementary function theory.

A less general and less precise decomposition of this sort was obtained by Alling [1]. In our notation and with the additional hypothesis that each $G_{j}$ have connected boundary, Alling showed that an $f \in \mathcal{O}(G)$ can be written in the form $f=f_{1}+\cdots+f_{q}+h$ where $f_{j} \in \mathcal{O}\left(X_{j}\right)$ and $h$ is some function holomorphic on a neighborhood of $\bar{G}$. Plainly no uniqueness assertion is possible for this decomposition.

A point of some interest in connection with the decomposition (2.1) is the observation, already noted by Alling, that if the function $f$ enjoys any of several restricted boundary behaviours then the functions $f_{1}, \cdots, f_{q}$ will exhibit the same boundary behaviour. Thus, e.g., if $f$ is bounded, then $f_{1}, \cdots, f_{q}$ will be bounded, if $f$ admits a continuous extension to $\bar{G}$, each $f_{j}$ will extend continuously to $\bar{X}_{j}$, etc.

Finally, let us observe that the uniqueness assertion of our theorem cannot be obtained, in general, without the hypotheses that the points $Q_{1}, \cdots, Q_{q}$ be non-Weierstrass points for $R$. This can be seen by very simple examples. Let $R$ be a hyperelliptic surface of genus at least five, and let $Q_{1}$ be a Weierstrass point of $R \cdot{ }^{3}$ Choose for $G$ a small annulus centered around $Q_{1}$ so that $R \backslash \bar{G}$ is the union of $G_{2}$, the "large" component, and $G_{1}$, the "small" component, a disc containing $Q_{1}$. Let $Q_{2}$ be any point of $G_{2}$. The Weierstrass gap sequence at $Q_{1}$ is $1,3, \cdots, 2 g-1$. A function $f$ meromorphic on $R$ with a pole of order four at $Q_{1}$ and no other singularities admits at least two decompositions of the form (2.1). First of all, if we take $f_{1}=f_{2}=0$ and $s=f$, we obtain a decomposition of the desired kind. Secondly, if $g$ is a meromorphic function with only one pole, that of order two and located at $Q_{1}$, we obtain a second decomposition of the desired form by taking $f_{2}=0, f_{1}=-g \mid X_{1}$, and $s=f+g$. (A discussion of Weierstrass points which goes far beyond what we need may be found in [2].)

We come now to the proof of the theorem.

Proof of THE THEOREM. Let $\mathcal{U}^{(1)}=\left\{U_{1}^{(1)}, U_{2}^{(2)}\right\}$ be the open cover for $R$ given by $U_{1}^{(1)}=G \cup \bar{G}_{1}, U_{2}^{(1)}=X_{1}$. The function $f$ is holomorphic in $G=U_{1}^{(1)} \cap U_{2}^{(1)}$ so by $\S \mathrm{I}$, there exist $f_{1} \in \mathcal{O}\left(X_{1}\right)$ and $f_{1}^{\prime} \in \mathcal{O}\left(G \cup \bar{G}_{1} ; Q_{1}^{-g}\right)$ such that $f=f_{1}\left|G+f_{1}^{\prime}\right| G$. By the Riemann-Roch theorem, there ex-

- Thus there exists in $\mathfrak{T}(R)$ a function with a single pole of order no more than $\boldsymbol{g}$ located at $Q_{1}$. 
ists a meromorphic function $m_{1} \in \mathcal{O}\left(R ; Q_{1}^{-0} Q_{2}^{-0}\right)$ such that if $f_{2}^{\prime \prime}=f_{1}^{\prime}-m_{1}$, then $f_{2}^{\prime \prime} \in \mathcal{O}\left(G \cup \bar{G}_{1}\right)$. We have that $f=f_{1}\left|G+f_{2}^{\prime \prime}\right| G+m_{1} \mid G$. If $\mathcal{u}^{(2)}$ $=\left\{U_{1}^{(2)}, U_{2}^{(2)}\right\}$ is the cover given by $U_{1}^{(2)}=G \cup \bar{G}_{1} \cup \bar{G}_{2}, U_{2}^{(2)}=X_{2}$, then by $\S I, f_{2}^{\prime \prime}=f_{2}\left|\left(G_{1} \cup G_{2}\right)+f_{2}^{\prime}\right|\left(G_{1} \cup G_{2}\right)$ where $f_{2} \in \mathcal{O}\left(U_{2}^{(2)}\right)$ and $f_{2}^{\prime}$ $\in \mathcal{O}\left(U_{2}^{(2)} ; Q_{2}^{-g}\right)$. By the Riemann-Roch theorem, there is $m_{2} \in \mathcal{O}(R$; $\left.Q_{1}^{-0} Q_{2}^{-g}\right)$ such that if $f_{3}^{\prime \prime}=f_{2}^{\prime}-m_{2}$, then $f_{3}^{\prime \prime} \in \mathcal{O}\left(U_{2}^{(2)}\right)$. This yields $f=f_{1}\left|G+f_{2}\right| G+f_{3}^{\prime \prime}\left|G+\left(m_{1}+m_{2}\right)\right| G$ with $f_{1} \in \mathcal{O}\left(X_{1}\right), f_{2} \in \mathcal{O}\left(X_{2}\right)$, and $f_{3}^{\prime \prime} \in \mathcal{O}\left(G \cup \bar{G}_{1} \cup G_{2}^{-}\right)$. If we repeat this process, we find after $q-1$ steps that

$$
f=\sum_{j=1}^{q-1} f_{j}\left|G+f_{q}^{\prime \prime}\right| G+\left(m_{1}+\cdots+m_{q-1}\right) \mid G
$$

where $f_{j} \in \mathcal{O}\left(X_{j}\right)$ and $f_{q}^{\prime \prime} \in \mathcal{O}\left(G \cup G_{1} \cup G_{2} \cup \ldots \cup G_{q-1}\right)=\mathcal{O}\left(X_{q}\right)$ and $m_{1}+m_{2}+\cdots+m_{q-1} \in \mathcal{O}\left(R ; Q_{1}^{-g} \cdots Q_{q}^{-g}\right)$. Thus, we have obtained a decomposition of the desired kind.

The uniqueness assertion of the theorem may be proved as follows. We assume that the points $Q_{j}$ are non-Weierstrass points and that we have decompositions

$$
\begin{aligned}
f & =f_{1}+\cdots+f_{q}+s \\
& =\tilde{f}_{1}+\cdots+\tilde{f}_{q}+\tilde{s}
\end{aligned}
$$

of the form (2.1) where each $f_{j}$ and $\tilde{f}_{j}$ vanishes at the point $P$ of $G$. The equations (2.2) imply that in $G$

$$
f_{1}-\tilde{f}_{1}=\sum_{j=2}^{q}\left(\tilde{f}_{j}-f_{j}\right)+\tilde{s}-s .
$$

The left side is in $\mathcal{O}\left(X_{1}\right)$ and vanishes at $P$, and the right side is in $\mathcal{O}\left(G \cup \bar{G}_{1} ; Q_{1}^{-o}\right)$. As $Q_{1}$ is a non-Weierstrass point, it follows that $f_{1}=\bar{f}_{1}$ and so that

$$
f_{2}-\bar{f}_{2}=\sum_{j=3}^{q}\left(\tilde{f}_{j}-f_{j}\right)+\bar{s}-s,
$$

and as $Q_{2}$ is a non-Weierstrass point, $f_{2}=\tilde{f}_{2}$. Repeating this process, we find that for each $j, f_{j}=\tilde{f}_{j}$ and then that $s=\tilde{s}$. This concludes the proof.

The second main result we will prove is the extension to compact Riemann surfaces of the Marcel Riesz theorem mentioned in the introduction. For this purpose we consider again the compact Riemann surface $R$ of genus $g$ and the domains $G$ and $G_{1}, \cdots, G_{q}$, but now it is necessary to impose additional regularity conditions on $\partial G$. We require that the pairwise disjoint simple closed curves which constitute $\Gamma$ be analytic so that $G$ is a regular region in the 
sense of [7]. To formulate our result, it is necessary to introduce a convenient measure on $\Gamma$. For this purpose fix a base point $P_{0} \in G$ and let $g\left(\cdot, P_{0}\right)$ be the Green's function for $G$ with singularity at $P_{0}$. If $\partial / \partial n$ denotes differentiation along the inner normal (with respect to $G$ ), we define the measure $\mu$ on $\Gamma$ by

$$
\int f d_{\mu}=\int_{\Gamma} f(P) \frac{\partial g\left(P, P_{0}\right)}{\partial n} d s \quad f \in \mathcal{C}(\Gamma)
$$

where $d s$ is the differential of arclength along $\Gamma$. All measure theoretic considerations on $\Gamma$ will refer to the measure $\mu$. The measure $\mu$ depends on the choice of $P_{0}$, but for various choices of $P_{0}$, the resulting measures are boundedly mutually absolutely continuous so for our purposes this dependence will be irrelevant, and we will suppress it in our notation. We will write $L^{p}(\Gamma)$ for the $L^{p}$-space associated with $\mu$. If $\Gamma_{j}$ denotes $\partial G_{j}$, then $\Gamma=\Gamma_{1} \cup \ldots \cup \Gamma_{q}$, and $\mu$ induces in an obvious way a measure on each $\Gamma_{j}$.

For $1 \leqq p<\infty$, for $D$ a domain in a Riemann surface, and for a divisor $\mathfrak{D}=P_{1}^{k_{1}} \ldots P_{m}^{k_{m}}$ on $D$ we define $H^{p}(D ; \delta)$ to be the space of those $f \in \mathcal{O}(D ; \delta)$ with the propeorty that $|f|^{p}$ has a harmonic majorant on $D \backslash K$ for some and therefore every compact neighborhood $K$ of the set $\left\{P_{1}, \cdots, P_{m}\right\}$. The theory of these spaces has been developed in detail, at least for domains $D$ with smooth boundary; it is set forth, e.g., in [3] and [6]. From this theory we will need the fact that if $D$ is one of the domains $G$ or $G_{j}$ and if $f \in H^{p}\left(D ; P^{k}\right)$, then for almost every point $Q \in \partial D$, the nontangential limit $f^{*}(Q)$ $=\lim _{Q^{\prime} \rightarrow Q} f\left(Q^{\prime}\right)$ exists and the function $f^{*}$ defined almost everywhere on $\partial D$ in this way is of class $L^{p}$. The functional

$$
f \mapsto\left(\int_{\partial D}\left|f^{*}\right| p d \mu\right)^{1 / p}
$$

is a norm on $H^{p}\left(D ; P^{k}\right)$, and with respect to this norm $H^{p}\left(D ; P^{k}\right)$ is a Banach space. We define $H^{p}\left(G_{1} \cup \ldots \cup G_{q}\right)$ to be

$$
\left\{f \in \mathcal{O}\left(G_{1} \cup \cdots \cup G_{q}\right): f \mid G_{j} \in H^{p}\left(G_{j}\right)\right\} .
$$

II.2. THEOREM. If $Q_{0}$ and $Q_{1}$ are points of $G$ and $R \backslash \bar{G}$ respectively and if $f \in L^{p}(\Gamma), 1<p<\infty$, there exist functions $f_{+} \in H^{p}(G), f_{-} \in H^{p}$ $\left(G_{1} \cup \ldots \cup G_{q}\right)$, and $s \in \mathcal{O}\left(Q_{0}^{-g} Q_{1}^{-o}\right)$ such that almost everywhere on $\Gamma$

$$
f=f_{+}^{*}+f_{-}^{*}+s \text {. }
$$

If neither $Q_{0}$ nor $Q_{1}$ is a Weierstrass point of $R$, this decomposition is unique provided we require that $f_{+}\left(S_{0}\right)=0$ for some fixed point $S_{0} \in G$ and $f_{-}\left(S_{1}\right)=0$ for some $S_{1} \in R \backslash \bar{G}$. 
In the case that $R$ is the Riemann sphere and $G$ is the open unit disc, we have $g=0$ so $\mathcal{O}\left(Q_{0}^{-o} Q_{1}^{-o}\right)=\boldsymbol{C}$ and the stated result is the familiar result of $M$. Riesz mentioned in the introduction. We will use Riesz's result in our proof; a discussion of it may be found in [5] or [8]. In the case that $R$ is the double of $G$ and the points $Q_{0}$ and $Q_{1}$ are conjugate under the canonical anticonformal map of $R$ which leaves $\Gamma$ fixed pointwise, the stated result is that established by Heins [3] except for an inessential difference in normalization.

Proof. The proof consists of two steps. First we observe that using the M. Riesz theorem we can, in a sense, solve the problem locally. Having this fact, the result of $\S I$ enables us to pass to a global solution. We obtain the uniqueness assertion by invoking a lemma proved by Royden.

Let the analytic simple closed curves which constitute $\Gamma_{k}$ be labelled $\gamma_{k, 1}, \cdots, \gamma_{k, \nu(k)}, k=1, \cdots, q$. For each $k=1, \cdots, q$, and each $n=1, \cdots, \nu(k)$, let $A(k, n)$ be a thin annulus about the curve $\gamma_{k, n}$, and let $A^{+}(k, n)=A(k, n) \cap G, A^{-}(k, n)=A(k, n) \cap G_{k}$. We choose the $A(k, n)$ so thin that they are pairwise disjoint. We also require that for some fixed annulus $B=\{z \in C: r<|z|<R\}, r<1<R$, there exist conformal homeomorphisms $\phi_{k, n}: B \rightarrow A(k, n)$ which carry the unit circle onto $\gamma_{k, n}$, which carry $B^{+}=\{z \in B:|z|<1\}$ onto $A^{+}(k, n)$, and which carry $B^{-}=\{z \in B:|z|>1\}$ onto $A^{-}(k, n)$. Let $U^{+}$denote the open unit disc and $U^{-}$the complement in the Riemann sphere of the closed unit disc. Finally, let $L^{p}(T)$ be the usual $L^{p}$-space associated with Lebesgue measure on the unit circle.

The function $f$ is in $L^{p}(\Gamma)$ so since $\phi_{k, n}$ is an analytic homeomorphism, the function $f \circ \phi_{k, n}$ lies in $L^{p}(T)$ and consequently there exist functions $f_{k, n}^{+} \in H^{p}\left(U^{+}\right)$and $f_{k, n}^{-} \in H^{p}\left(U^{-}\right)$such that almost everywhere on $T$

$$
f \circ \phi_{k, n}=\left(f_{k, n}^{+}\right)^{*}+\left(\overline{f_{k, n}^{-}}\right)^{*} .
$$

Carrying the functions $f_{k, n}^{+}$and $f_{k, n}^{-}$up to $R$ by way of $\phi_{k, n}^{-1}$, we see that if we define $A^{+}$by

$$
A^{+}=\bigcup\left\{A^{+}(k, n): k=1, \cdots, q ; n=1, \cdots, \nu(k)\right\}
$$

and $A_{\bar{k}}^{-}, k=1, \cdots, q$ by

$$
A_{\bar{k}}^{-}=\bigcup\left\{A^{-}(k, n): n=1, \cdots, \nu(k)\right\},
$$

then there exist functions $f_{0}^{+} \in H^{p}\left(A^{+}\right)$and $f_{k} \in H^{p}\left(A_{\mathbf{k}}^{-}\right)$such that, almost everywhere on $\Gamma_{k}$,

$$
f=f_{0}^{*}+f_{k}^{*}
$$


Thus we have solved the problem locally; it remains to pass to the global solution by the cohomological tool of $\S \mathrm{I}$. For this step, it is convenient to assume that the indexing is such that $Q_{1} \in G_{1}$.

Let $\mathfrak{U}_{k}$ be the open cover $\left\{G_{k}, V_{k}\right\}$ for $R$ where

$$
V_{k}=X_{k} \cup \cup\{A(k, n): n=1, \cdots, \nu(k)\} .
$$

We have $G_{k} \cap V_{k}=A_{k}^{-}$so $f_{k} \in \mathcal{O}\left(G_{k} \cap V_{k}\right)$ and it follows that for some choice of functions $f_{k}^{-} \in \mathcal{O}\left(G_{k} ; Q_{1}^{-g}\right)$ and $f_{k}^{+} \in \mathcal{O}\left(V_{k}\right)$ if $k=1$ and $f_{k}^{-} \in \mathcal{O}\left(G_{k}\right)$, $f_{k}^{+} \in \mathcal{O}\left(V_{k} ; Q_{1}^{-o}\right)$ if $k>1$, we have that on $A_{k}^{-}$

$$
f_{k}=\bar{f}_{k}-f_{k}^{+} \text {. }
$$

As $f_{k} \in H^{p}\left(A_{k}^{-}\right)$and $f_{k}^{+}$is holomorphic on a neighborhood of $\Gamma_{k}$, we see that $f_{k}^{-} \in H^{p}\left(G_{k}\right),(k>1)$, and $f_{1}^{-} \in H^{p}\left(G_{1} ; Q_{1}^{-o}\right)$. Similarly, there exist functions $f_{0}^{+} \in H^{p}(G)$ and $f_{0}^{-} \in \mathcal{O}\left(G_{1} \cup \ldots \cup G_{q} \cup \cup A(n, k) ; Q_{1}^{-\vartheta}\right)$ with the property that in $A^{+}$

$$
f_{0}=\bar{f}_{0}-f_{0}^{+} \text {. }
$$

We define functions $F_{+}$on $G$ and $F_{-}$on $G_{1} \cup \cdots \cup G_{q}$ by requiring that

$$
F_{+}=-\left(f_{0}^{+}+f_{1}^{+}+\cdots+f_{q}^{+}\right)
$$

and that on $G_{k}$

$$
F_{-}=\overline{f_{0}}+f_{1}^{+}+\cdots+f_{k-1}^{+}+\overline{f_{k}}+\hat{f}_{k+1}^{+}+\cdots+\hat{f}_{q}^{+} .
$$

The function $F_{+}$lies in $H^{p}(G)$ and $F_{-}$lies in $H^{p}\left(G_{1} \cup \ldots \cup G_{q} ; Q_{1}^{-g}\right)$. On $\Gamma_{k}$ we have almost everywhere

$$
\begin{aligned}
F_{+}^{*}+F_{-}^{*} & =\overline{f_{0}^{*}}-f_{0}^{+*}+\overline{f_{k}^{*}}-f_{k}^{+*} \\
& =f_{0}^{*}+f_{k}^{*} \\
& =f .
\end{aligned}
$$

There exists a constant $m_{+}$and, by the Riemann-Roch theorem, an element $m_{-} \in \mathcal{O}\left(Q_{0}^{-g} Q_{1}^{-g}\right)$ such that if $f_{+}=F_{+}-m_{+}$and $f_{-}=F_{-}-m_{-}$, then $f_{+}$is holomorphic in $G$ and vanishes at $S_{0}$ and $f_{-}$is holomorphic in $G_{1} \cup \ldots \cup G_{q}$ and vanishes at $S_{1}$. If we put $s=m_{+}+m_{-}$, we have, almost everywhere on $\Gamma$

$$
f=f_{+}^{*}+f_{-}^{*}+s,
$$

a decomposition of the desired kind.

To prove uniqueness, assume that in addition to (2.3), we have a decomposition 


$$
f=g_{+}^{*}+g_{-}^{*}+t
$$

of the kind described in the theorem, and suppose that neither of $Q_{0}$ nor $Q_{1}$ is a Weierstrass point for $R$. We have then that

$$
f_{+}^{*}-g_{+}^{*}=g_{-}^{*}-f_{-}^{*}+t-s .
$$

As $g_{-}-f_{-}+t-s$ belongs to the class $H^{p}\left(G_{1} \cup \ldots \cup G_{q} ; Q_{1}^{-0}\right)$ and $f_{+}-g_{+}$belongs to $H^{p}(G)$, a result of Royden [6, Lemma 7] yields the fact that these functions are analytic continuations of each other across $\Gamma$. As $Q_{1}$ is a non-Weierstrass point, both sides must be constant, and since $f_{+}\left(S_{0}\right)=0=g_{+}\left(S_{0}\right)$, we conclude that $f_{+}=g_{+}$and then that on $\Gamma$

$$
f_{-}^{*}-g_{-}^{*}=t-s .
$$

Again, this yields the fact that $f_{-}=g_{-}$and hence that $s=t$. This concludes the proof.

We conclude with a remark on technique. We have chosen to employ the result on principal $\boldsymbol{C}$-bundles quoted in $\S \mathrm{I}$. We could obtain both our results by invoking rather the theory of the Cauchy integral on compact Riemann surfaces as developed in [4], but this approach would be technically cumbersome at various points along the way. Of course it will be recognized that the Cauchy kernel has been used indirectly: Röhrl uses it in [4] to establish the existence of sections of principal $\mathbf{C}$-bundles.

\section{REFERENCES}

1. N. L. Alling, Extensions of meromorphic function rings over non-compact Riemann surfaces. II, Math. Z. 93 (1966), 345-394. MR 33 \#7572.

2. R. C. Gunning, Lectures on Riemann surfaces, Princeton Mathematical Notes, Princeton Univ. Press, Princeton, N. J., 1966. MR 34 \#7789.

3. M. Heins, Symmctric Riemann surfaces and boundary problems, Proc. London Math. Soc. (3) 14a (1965), 129-143. MR 35 \#4400.

4. A. Hurwitz and R. Courant, Funktionentheorie, 4th ed., with an appendix by H. Röhrl, Springer-Verlag, Berlin, 1964. MR 30 \#3959.

5. M. Riesz, Sur les fonctions conjugés, Math. Z. 27 (1927), 218-244.

6. H. L. Royden, The boundary values of analytic and harmonic functions, Math. $Z$. 78 (1962), 1-24. MR $25 \# 2190$.

7. G. Springer, Introduction to Riemann surfaces, Addison-Wesley, Reading, Mass., 1957. MR 19, 1169.

8. A. Zygmund, Trigonometric Series. Vol. I, Cambridge Univ. Press, New York, 1959. MR $21 \# 6498$.

YALE UNIVERSITY 